\title{
A Single Center Experience with Early Adoption of Physiologic Pacing Approaches
}

\author{
Connor Oates ${ }^{1}$, Iwanari Kawamura ${ }^{1}$, Mohit turagam $^{1}$, Marie-Noelle Langan ${ }^{1}$, Mary \\ McDonaugh $^{1}$, William Whang ${ }^{1}$, Marc Miller ${ }^{1}$, Daniel Musikantow ${ }^{1}$, Srinivas Dukkipati ${ }^{1}$, \\ Vivek Reddy ${ }^{1}$, and Jacob Koruth ${ }^{1}$ \\ ${ }^{1}$ Icahn School of Medicine at Mount Sinai
}

September 25, 2021

\begin{abstract}
Background: Increasing interest in physiological pacing has been countered with challenges such as accurate lead deployment and increasing pacing thresholds with His-bundle pacing (HBP). More recently, left bundle branch area pacing (LBBAP) has emerged as an alternative approach to physiologic pacing. Objective: To compare procedural outcomes and pacing parameters at follow-up during initial adoption of HBP and LBBAP at a single center. Methods: Retrospective review, from September 2016 to January 2020, identified the first 50 patients each who underwent successful HBP or LBBAP. Pacing parameters were then assessed at first follow-up after implantation and after approximately one year, evaluating for acceptable pacing parameters defined as sensing R-wave amplitude $>5 \mathrm{mV}$, threshold $<2.5 \mathrm{~V} @ 0.5 \mathrm{~ms}$ and impedance between 400 and 1200 Ohms. Results: The HBP group was younger with lower ejection fraction compared to LBBP ( $73.2 \pm 15.3$ vs $78.2 \pm 9.2$ years, $\mathrm{p}=0.047 ; 51.0 \pm 15.9 \%$ vs $57.0 \pm 13.1 \%, \mathrm{p}=0.044)$. Post-procedural QRS widths were similarly narrow $(119.8 \pm 21.2$ vs. $116.7 \pm 15.2 \mathrm{~ms} ; \mathrm{p}=0.443)$ in both groups. Significantly fewer patients with HBP met the outcome for acceptable pacing parameters at initial follow-up (56.0\% vs $96.4 \%, \mathrm{p}=0.001)$ and most recent follow-up $(60.7 \%$ vs $94.9 \%, \mathrm{p}=<0.001$; at $399 \pm 259$ vs. $228 \pm 124$ days, $\mathrm{p}=<0.001)$. More HBP patients required lead revision due to early battery depletion ( 0 vs $13.3 \%$, at an average of 664 days). Conclusion: During initial adoption, as compared with LBBAP, HBP is associated with a significantly higher frequency of unacceptable pacing parameters, energy consumption, and lead revisions.
\end{abstract}

\section{A Single Center Experience with Early Adoption of Physiologic Pacing Approaches}

Running title: Early Adoption of Physiological Pacing

Connor P. Oates, MD, Iwanari Kawamura, MD, Mohit K. Turagam, MD, Marie-Noelle Langan, MD, Mary McDonaugh MS, NP William Whang, MD, Marc A. Miller, MD, Daniel R. Musikantow, MD, Srinivas R. Dukkipati, MD, Vivek Y. Reddy, MD and Jacob S. Koruth, MD

Helmsley Electrophysiology Center, Department of Cardiology, Icahn School of Medicine at Mount Sinai, New York, NY, USA

Word count: 2516

Tables: 3

Figures: 3

Funding: None

\section{Corresponding Author}

Jacob S. Koruth, MD 
Icahn School of Medicine at Mount Sinai

One Gustave L. Levy Place, Box 1030, New York, NY, 10029

Tel: (212)241-7114 Fax: (646)537-9691

E-mail: jacob.koruth@mountsinai.org

\section{Disclosures}

Vivek Reddy : Abbott (Consultant), Ablacon (Consultant, Equity), Acutus Medical (Consultant, Equity), Affera (Consultant, Equity), Apama Medical (Consultant, Equity), Aquaheart (Consultant, Equity), Autonomix (Consultant, Equity), Axon (Consultant), Backbeat (Consultant, Equity), BioSig (Consultant, Equity), Biosense-Webster (Consultant), Biotronik (Consultant), Boston Scientific (Consultant), Cardiofocus (Consultant), Cardionomic (Consultant), CardioNXT / AFTx (Consultant), Circa Scientific (Consultant, Equity), Corvia Medical (Consultant, Equity), East End Medical (Consultant, Equity), EBR (Consultant), EPD (Consultant, Equity), Epix Therapeutics (Consultant, Equity), EpiEP (Consultant, Equity), Eximo (Consultant, Equity), Farapulse (Consultant, Equity), Fire1 (Consultant, Equity), Impulse Dynamics (Consultant), Javelin (Consultant, Equity), Keystone Heart (Consultant, Equity), LuxCath (Consultant, Equity), Manual Surgical Sciences (Equity), Medlumics (Consultant, Equity), Medtronic (Consultant), Middlepeak (Consultant, Equity), Newpace (Equity), Nuvera (Consultant, Equity), Philips (Consultant), Stimda (Consultant), Surecor (Equity), Thermedical (Consultant), Valcare (Consultant, Equity), Vizara (Equity) and VytronUS (Consultant, Equity).

Jacob Koruth Equity: Affera, Consultant- Abbott, VytronUS, Cardiofocus, Farapulse, Research grantsVytronUS, Cardiofocus, Luxcath, Biosense Webster, Farapulse, Affera

All other authors report no relevant disclosures

\section{Abstract:}

Background : Increasing interest in physiological pacing has been countered with challenges such as accurate lead deployment and increasing pacing thresholds with His-bundle pacing (HBP). More recently, left bundle branch area pacing (LBBAP) has emerged as an alternative approach to physiologic pacing.

Objective : To compare procedural outcomes and pacing parameters at follow-up during initial adoption of HBP and LBBAP at a single center.

Methods : Retrospective review, from September 2016 to January 2020, identified the first 50 patients each who underwent successful HBP or LBBAP. Pacing parameters were then assessed at first follow-up after implantation and after approximately one year, evaluating for acceptable pacing parameters defined as sensing R-wave amplitude $>5 \mathrm{mV}$, threshold $<2.5 \mathrm{~V} @ 0.5 \mathrm{~ms}$ and impedance between 400 and 1200 Ohms.

Results : The HBP group was younger with lower ejection fraction compared to LBBP (73.2 \pm 15.3 vs $78.2 \pm 9.2$ years, $\mathrm{p}=0.047 ; 51.0 \pm 15.9 \%$ vs $57.0 \pm 13.1 \%, \mathrm{p}=0.044)$. Post-procedural QRS widths were similarly narrow $(119.8 \pm 21.2$ vs. $116.7 \pm 15.2 \mathrm{~ms} ; \mathrm{p}=0.443)$ in both groups. Significantly fewer patients with HBP met the outcome for acceptable pacing parameters at initial follow-up (56.0\% vs $96.4 \%, \mathrm{p}=0.001)$ and most recent follow-up ( $60.7 \%$ vs $94.9 \%, \mathrm{p}=<0.001$; at $399 \pm 259$ vs. $228 \pm 124$ days, $\mathrm{p}=<0.001)$. More HBP patients required lead revision due to early battery depletion ( 0 vs $13.3 \%$, at an average of 664 days).

Conclusion : During initial adoption, as compared with LBBAP, HBP is associated with a significantly higher frequency of unacceptable pacing parameters, energy consumption, and lead revisions.

\section{Keywords}

Physiologic pacing; left bundle branch area pacing; His bundle pacing; pacemaker; early adoption; thresholds; lead revision

\section{Introduction}


Right ventricular (RV) apical pacing has remained the standard approach to ventricular pacing despite evidence that it causes electrical dyssynchrony associated with an increased risk of developing atrial fibrillation and left ventricular systolic dysfunction. ${ }^{1-4}$ The current popularity of RV apical pacing as the preferred pacing site is driven primarily by the ease of placing pacing leads into the apex, as well as the stability of pacing parameters at this location. In recent years, "physiological" approaches to pacing have attracted significant interest as electrophysiologists seek to maintain left ventricular synchrony and mitigate the adverse effects of RV apical pacing. Though His-bundle pacing (HBP) had emerged as a promising approach to physiologic pacing, HBP can be technically challenging to perform using current tools. ${ }^{5-10}$ Furthermore, HBP has been associated with a high incidence of rising pacing thresholds and low sensing values. Indeed, unacceptable pacing parameters over time can mandate lead revision and/or generator replacement for pre-mature battery depletion. Together, these serve as major deterrents to the adoption of physiologic pacing when compared to traditional RV apical pacing. ${ }^{11-14}$

More recently, left bundle branch area pacing (LBBAP) has emerged as an attractive alternative to achieving physiologic pacing - particularly since initial studies have not reported rising pacing thresholds or reduced sensing values. ${ }^{15-19}$ As operators begin to explore physiologic pacing strategies in their practice, it is unclear whether one should pursue HBP, LBBAP or a combination of the two strategies. In this study, we compared procedural outcomes and intermediate follow-up for the first 50 patients at our institution undergoing either HBP or LBBP. By sharing this early experience with both approaches to physiological pacing, we aim to highlight the challenges one may experience with the adoption of physiological pacing.

\section{Methods}

\section{Study Design :}

We conducted a retrospective, observational study of consecutive patients who underwent physiological pacing (either HBP or LBBAP) between September 2016 and September 2020, at one large academic medical center. The first 50 patients from each group who underwent successful implantation procedures were identified and included in this analysis. All patients underwent pacemaker implantation for standard indications consistent with current guidelines. This study was approved by the Institution Review Board at Mount Sinai Hospital.

\section{Implant procedure:}

HBP was performed using the 3830 SelectSecure lead (Medtronic, Minneapolis, MN) delivered through either the fixed curve (C315-HIS) or deflectable curve (C304) sheath. Standard techniques for HBP were used, including mapping for the distal His recording prior to fixation or pace-mapping when the His recording was not located (infrequently). The procedure was deemed successful if selective or nonselective His-bundle capture was demonstrable. RV apical pacing leads were also implanted in $12 \%$ (6/50) of patients receiving HBP with the apical lead programmed to pace $80 \mathrm{~ms}$ after His-pacing. At implant, pacing outputs were generally programmed at $3.5 \mathrm{~V}$ at $1 \mathrm{~ms}$ and then adjusted based on the HBP capture threshold (confirmed by 12 lead EKG morphology) during follow-up, such that outputs were programmed to twice the capture threshold for His-capture.

LBBAP was also performed using the above lead and sheaths. Standard techniques were used, as previously described. ${ }^{15}$ Briefly, the lead was advanced $2 \mathrm{~cm}$ distal to the His electrogram toward the RV apex or on the basis of unipolar paced morphology. ${ }^{20}$ With the sheath held flush against the septum, the lead was rotated and advanced until the lead perforated the RV septal myocardium. The paced QRS morphology and impedance was continuously monitored and the lead rotated until the paced morphology approximate a RBBB morphology. Contrast injection was performed in select cases. At implant, outputs were generally programmed at 2-2.5 $\mathrm{V}$ at $0.5 \mathrm{~ms}$, and then adjusted only if needed during follow-up based on threshold (confirmed by 12 lead EKG morphology) such that outputs were programmed to twice that of threshold.

\section{Data Collection and Follow-Up :}

Baseline demographic, clinical, electrocardiographic, and procedural data were collected by chart review. 
Follow-up data from the first post-procedure follow-up and from the most recent follow-up were collected for all patients. Pacing threshold was defined as the lowest voltage required to capture the conduction system with either selective or nonselective morphology. Pacing parameters were recorded in detail with 12-lead ECG performed during threshold testing. Pacing thresholds, R-wave sensing and impedances were all carefully documented at implant and in follow-up. Stimulus to peak R wave in lead V6 (RWPT) was defined as the time in milliseconds between the pacing stimulus and the peak of the R-wave in V6 on the surface electrocardiogram. Pacemaker stimulation energy was analyzed as a function of voltage and pulse width $\left(\mathrm{E}=\mathrm{V}^{2} \mathrm{t} / \mathrm{R}\right.$ where $\mathrm{E}=$ energy, $\mathrm{V}=$ voltage, $\mathrm{t}=$ pulse width and $\mathrm{R}=$ impedance $){ }^{14}$ Though pulse widths of $0.4,0.5$ and $1 \mathrm{~ms}$ were used variably across the population, we were able to use this method to adjust thresholds to reflect an amplitude measured at a pulse width of $0.5 \mathrm{~ms}$ for consistency across all measurements (adjusted pulse amplitude $=\operatorname{sqrt}\left[\left(\right.\right.$ recorded pulse amplitude) ${ }^{2}$ (recorded pulse width) $\left./(0.5)\right]$. For the purpose of analysis, an 'acceptable pacing endpoint' (APE) included the following parameters: sensing R-wave amplitude $>5 \mathrm{mV}$, pacing threshold $<2.5 \mathrm{~V}$ @ $0.5 \mathrm{~ms}$ and impedance between 400 and 1200 Ohms. APE captures normal lead behavior as seen with legacy pacing. Total fluoroscopy duration and procedure duration was obtained. Follow-up for each patient stopped with any lead revision or generator replacement.

\section{Statistical Analysis :}

Pre-procedural, procedural and follow-up data for patients who underwent HBP were compared with patients who underwent LBBP. Continuous variables were reported as mean +/- SD and compared with two-sample ttests. Categorical variables were reported as percentages and compared using chi squared or two-tailed Fisher exact tests as appropriate. The Kaplan-Meier method was used to generate failure curves for descriptive purposes with censoring performed at either the date of loss of APE, date of last follow-up, or date of death. All analyses were performed with the use of Stata software version 16.1. Statistical significance was defined by p-values $<0.05$.

\section{Results}

\section{Baseline characteristics}

Patients underwent pacemaker implantation for sick sinus syndrome, high degree atrioventricular block, complete heart block and/or cardiac resynchronization therapy. There were no significant differences amongst indications in HBP vs LBBAP groups. Equal numbers of patients in both groups (20\%) developed indications for pacing following transcatheter aortic valve replacement. Patients undergoing HBP were younger than those who underwent LBBAP (73.2 \pm 15.3 vs $78.2 \pm 9.2$ years, $\mathrm{p}=0.047)$. Though there was a non-significant trend towards more patients in the HBP group with ischemic or non-ischemic cardiomyopathy, the HBP group had a lower LV ejection fraction compared to the LBBAP group ( $51.0 \pm 15.9$ vs $57.0 \pm 13.1 \%, \mathrm{p}=0.044)$. There was no significant difference between pre-procedural intrinsic QRS width $(135.4 \pm 29.1$ vs. $131.0 \pm 34.6 \mathrm{~ms}, \mathrm{p}$ $=0.505)$, incidence of right bundle branch block or left bundle branch block $(37.5$ vs. $42.0 \%, \mathrm{p}=0.649 ; 25.0$ vs. $22.0 \%, \mathrm{p}=0.726$ ) between groups (Table 1 ).

\section{Procedural characteristics}

All patients included in this study underwent clinically successful implants. HBP took significantly longer and required significantly more fluoroscopy compared to LBBAP (124.7 \pm 50.4 vs. $105 \pm 28.8$ minutes, $\mathrm{p}=$ $0.023 ; 19.8 \pm 15.9$ vs. $13.7 \pm 7.4$ minutes; $\mathrm{p}=0.019)$. These differences persisted even after excluding cases (4 HBP cases and 1 LBBAP case) in which physiologic pacing was used as a bailout option after inadequate resynchronization therapy with a coronary sinus lead (118.7 \pm 48.0 vs. $103.7 \pm 27.8$ minutes, $\mathrm{p}=0.037$; $16.8 \pm 12.2$ vs. $13.1 \pm 6.2$ minutes; $\mathrm{p}=0.036$ ).

The post-implant paced QRS width in the HBP and LBBAP groups were similarly narrow (119.8 \pm 21.2 vs.116.7 $\pm 15.2 \mathrm{~ms}, \mathrm{p}=0.443)$ with a $19.8 \%$ and $17.2 \%$ reduction of QRS width from baseline, respectively. Stimulus to peak R wave in lead V6 (RWPT) times were not significantly different in patients undergoing HBP and LBBAP ( $80 \pm 22.9$ vs. $76.4 \pm 14.5 \mathrm{~ms}, \mathrm{p}=0.370$ ). Post-procedurally, $86.7 \%$ of the LBBAP group achieved APE, while only $68.8 \%$ of the HBP group met the APE (Figure 1). This difference was not statistically 
significant. When analyzed independently, however, the pacing threshold was significantly higher in patients undergoing HBP $(1.1 \pm 0.9$ vs. $0.5 \pm 0.3 \mathrm{~V}, \mathrm{p}<0.001)$ and R-wave amplitude and impedance were significantly lower in patients undergoing HBP compared to LBBAP ( $8.7 \pm 6.1$ vs. $11.9 \pm 5.7 \mathrm{mV}, \mathrm{p}=0.035 ; 615.3 \pm 209.8$ vs. $715 \pm 154.2$ Ohms, $\mathrm{p}=0.012$ ) (Figures 2 ). There were no immediate procedure-related complications noted in either group

\section{Follow-up}

First follow-up occurred at 43.4 and 44.0 days in the HBP and LBBAP groups, respectively $(\mathrm{p}=0.94)$. At this follow-up, $40 \%$ fewer patients receiving HBP met the APE compared to patients receiving LBBAP (56 vs $96.4 \%, \mathrm{p}=0.001)$. Unlike in patients with LBBAP, pacing thresholds increased from post-implantation to first follow-up in patients with HBP. Pacing thresholds were significantly higher $(1.4 \pm 1.1$ vs. $0.7 \pm 0.3 \mathrm{~V}$, $\mathrm{p}<0.001)$ while R-wave amplitudes and impedance remained significantly lower $(8.6 \pm 6.4 \mathrm{vs} .16 .0 \pm 4.9 \mathrm{mV}$, $\mathrm{p}<0.001 ; 454 \pm 118.4$ vs. $540.3 \pm 59.6$ Ohms, $\mathrm{p}<0.001)$ in the HBP group compared to the LBBAP group.

Most recent follow-up was significantly longer for the HBP compared to the LBBAP group (398.8 \pm 259.3 vs. $228.0 \pm 124.3$ days, $\mathrm{p}=<0.001$ ). At most recent follow-up, the HBP group demonstrated a persistently lower rate of APE compared to the LBBAP group (60.7\% vs $94.9 \%, \mathrm{p}<0.001)$. Compared with the HBP group, the LBBAP group had a significantly greater proportion of survival with APE $(\mathrm{p}<0.001)$ (Figure 3 ). Threshold amplitudes remained significantly higher in patients with HBP compared to LBBAP $(2.1 \pm 2.2$ vs. $0.8 \pm 0.3 \mathrm{~V}, \mathrm{p}<0.001$ ), whereas $\mathrm{R}$-wave amplitudes and impedances continued to be significantly lower after HBP $(8.9 \pm 6.6$ vs. $16.1 \pm 4.5 \mathrm{mV}, \mathrm{p}<0.001 ; 434.2 \pm 108.1$ vs. $535.5 \pm 56.8$ Ohms, $\mathrm{p}<0.001)$. During the study period, $13.3 \%$ of patients in the HBP group required lead revision and generator replacement due to early battery depletion at a mean of 664.3 days.

\section{Discussion}

Our report focuses on comparing the procedural outcomes of two current approaches to "physiological" pacing during the initial learning curve phase of implanting physicians at a single center. By describing this early experience with both forms of pacing, we hope to better inform other adopters as they consider both these options.

In this report, we observed the following: 1) His-bundle and left bundle branch area pacing result in similarly narrow paced QRS widths [?] $120 \mathrm{~ms}(61.9 \%$ vs $65.9 \%, \mathrm{p}=0.62)$ and can be achieved with a low risk of acute complications. 2) Significant deterioration in pacing parameters emerged as soon as 4 weeks after implant in the HBP group and persisted/worsened over follow up. This pattern was not seen in the LBBAP group. 3) A significantly greater rate of adverse events (lead revisions and premature generator change) occurred in the HBP group (13\%) compared to the LBBAP group (0\%).

Immediately following implantation, the acceptable pacing endpoint (APE) was met by $18 \%$ fewer patients in the HBP group compared to the LBBAP group. This measure of lead safety and efficiency progressively worsened over time. By first follow-up, $40.4 \%$ fewer patients continued to meet the APE in the HBP group compared to LBBAP. This marked difference persisted to the most-recent follow-up. The deterioration of pacing parameters was driven primarily by worsening pacing thresholds, culminating in 6 of 45 HBP patients $(13.3 \%)$ requiring lead revision and generator replacements at follow-up. R-wave amplitudes and lead impedance remained stable with additional follow-up in both groups.

Experienced implanters place leads into the RV apex with relative ease. Despite early procedural complications like lead dislodgement and perforation, progressive threshold rises rarely occur and apical pacing remains the traditional RV pacing site of choice. ${ }^{21}$ In contrast, the $13 \%$ of patients in the HBP group who required lead revisions in this report highlights a distinct shortcoming of choosing the His-bundle as the site for physiological pacing as opposed to LBBAP.

The incidence of progressive rise in thresholds and lead revision rates amongst early adopters of HBP has been variable in the literature. ${ }^{22-23}$ Bhatt et al, Keene et al and Teigeler et al in their respective single center reports described $8 \%, 7.5 \%$ and $11 \%$ rates of lead revision/intervention, respectively. ${ }^{11,14,24}$ 
On the other hand, Chaumont et al. reported a much lower incidence of lead revisions in their multicenter experience (3.4\%) and Qian et al. reported no lead revisions. ${ }^{25-26}$ The threshold rises have been postulated to be the result of several mechanisms: 1) a relative lack of muscle in the underling region of the Hisbundle 2) progressive fibrotic changes that occur over time after lead fixation, and 3) progressive degrees of micro-dislodgement. ${ }^{16,27}$ On the other hand, while published reports of LBBAP pacing have been primarily restricted to centers with significant technical expertise, reports of significant threshold changes and lead revisions are distinctly uncommon $(<1 \%)$ in patients undergoing LBBAP. $^{20,28-29}$

In their single center comparative study of HBP and LBBAP, Qian et al did not report any lead revisions, while noting an increase in capture threshold in $12.5 \%$ of patients in the HBP group. ${ }^{26}$ In contrast, our single center comparative report describes a $13 \%$ rate of lead revision/generator change in the HBP group. Importantly, our events all occurred beyond the one-year of follow-up period, beyond the time studied by Qian et al. Our study underscores how, with continued follow-up of patients with HBP, progressive worsening of pacing parameters over time clinically impacts the lives of our patients.

Our $13 \%$ event rate of lead revisions is higher than many of the above-mentioned reports and may reflect differences in our implant technique: for example, we did not consistently document His-bundle injury during the procedure, which has been described as predictive of lower chronic thresholds. While the early nature of our experience may partly explain these rates, they are in stark contrast to our LBBAP group where no patients experienced any additional procedures during follow-up. The comparatively shorter period of followup of our LBBAP group is a limitation. But at most recent follow-up, the LBBAP group has not shown any deterioration in pacing parameters, whereas the HBP group had already demonstrated a deterioration in APE at the same comparable point of follow-up.

While all LBBAP implanters in this study benefited from the learning curve of HBP, the differences in outcomes between both groups suggest distinct advantages of placing the lead deep within the septum. In fact, while early lead dislodgement and, rarely, lead perforations in the LV cavity can occur in LBBAP, threshold rises have not been reported using this technique and were not seen in our experience. Though it is likely that inexperienced operators will fail at consistently achieving selective or non-selective LBB capture (i.e., only left ventricular septal capture), the necessity of actual LBB capture is unclear and the almost obligate increases in pacing thresholds are not seen. ${ }^{30}$ LBBAP leads to very low lead revision/premature generator replacement rates with no incremental procedural risk for dependent patients. These should be requisite characteristics for the widespread adoption of any alternative to RV apical pacing.

\section{Study Limitations :}

This report describes the experience of a select few operators at a single institution. During this study, operators transitioned from pursuing HBP to LBBAP in an almost sequential fashion. The initial experience with HBP undoubtedly had a positive impact on certain procedural aspects of LBBAP such as fluoroscopy and procedural times. Importantly, this study did not systematically evaluate for the presence of LBB capture in the LBBAP group.

Table 1: Baseline Characteristics of Patients Undergoing Physiologic Pacing

\begin{tabular}{llll}
\hline & His Pacing $(\mathbf{n}=50)$ & LBBA Pacing $(\mathbf{n}=50)$ & P-value \\
\hline Age (mean) & $73.2 \pm 15.3$ & $78.2 \pm 9.2$ & $\mathrm{p}=0.047$ \\
Male (\%) & $28 / 50(56.0 \%)$ & $31 / 50(61.8 \%)$ & $\mathrm{p}=0.542$ \\
LVEF (\%) & $51.0 \pm 15.9$ & $57.0 \pm 13.1$ & $\mathrm{p}=0.044$ \\
Ischemic Cardiomyopathy & $6 / 50(12.0 \%)$ & $1 / 50(2.0 \%)$ & $\mathrm{P}=0.050$ \\
Non-Ischemic Cardiomyopathy & $16 / 50(32.0 \%)$ & $8 / 50(16.0 \%)$ & $\mathrm{p}=0.061$ \\
Intrinsic QRS (ms) & $135.4 \pm 29.1$ & $131.0 \pm 34.6$ & $\mathrm{p}=0.505$ \\
Left Bundle Branch Block & $12 / 50(25.0 \%)$ & $11 / 50(22.0 \%)$ & $\mathrm{p}=0.726$ \\
Right Bundle Branch Block & $18 / 50(37.5 \%)$ & $21 / 50(42.0 \%)$ & $\mathrm{p}=0.649$ \\
Post-TAVR & $10 / 50(20.0 \%)$ & $10 / 50(20.0 \%)$ & $\mathrm{p}=1.000$
\end{tabular}




\begin{tabular}{llll}
\hline & His Pacing $(\mathbf{n = 5 0})$ & LBBA Pacing $(\mathbf{n}=\mathbf{5 0})$ & P-value \\
\hline Pacemaker Indications & & & \\
Sick sinus syndrome & $8 / 50(16.0 \%)$ & $6 / 50(12.0 \%)$ & $\mathrm{p}=0.564$ \\
High degree AV Block & $15 / 50(30.0 \%)$ & $20 / 50(40.0 \%)$ & $\mathrm{p}=0.564$ \\
Complete AV block & $19 / 50(38.0 \%)$ & $21 / 50(42.0 \%)$ & $\mathrm{p}=0.683$ \\
Resynchronization & $8 / 50(16.0 \%)$ & $3 / 50(6.0 \%)$ & $\mathrm{p}=0.110$ \\
\hline
\end{tabular}

Table 2: Procedural Outcomes and Follow-Up Characteristics of Patients Undergoing Physiologic Pacing

\begin{tabular}{llll}
\hline Procedural characteristics & His Pacing & LBBA Pacing & P-value \\
\hline Intrinsic QRS (ms) & $135.4 \pm 29.1$ & $131.0 \pm 34.6$ & $\mathrm{p}=0.505$ \\
Post-implant paced QRS (ms) & $119.8 \pm 21.2$ & $116.7 \pm 15.2$ & $\mathrm{p}=0.443$ \\
Reduction in QRS Duration & $0.86 \pm 0.18$ & $0.91 \pm 0.23$ & $\mathrm{p}=0.314$ \\
Follow up QRS Duration (ms) & $120.1 \pm 20.5$ & $115.9 \pm 13.5$ & $\mathrm{p}=0.336$ \\
Reduction in QRS Duration & $0.87 \pm 0.19$ & $0.91 \pm 0.24$ & $\mathrm{p}=0.519$ \\
QRS [?] 120 ms & $26 / 42(61.9 \%)$ & $29 / 44(65.9 \%)$ & $\mathrm{p}=0.622$ \\
R-wave peak time (ms) & $80 \pm 22.9$ & $76.4 \pm 14.5$ & $\mathrm{p}=0.370$ \\
Procedure duration (min) & $124.7 \pm 50.4$ & $105 \pm 28.8$ & $\mathrm{p}=0.023$ \\
Fluoroscopy time (min) & $19.8 \pm 15.9$ & $13.7 \pm 7.4$ & $\mathrm{p}=0.019$ \\
Pacing Parameters & & & \\
Post procedure & & & \\
Threshold Amplitude (V @ 0.5 ms) & $1.1 \pm 1.0$ & $0.54 \pm 0.3$ & $\mathrm{p}<0.001$ \\
R-Wave Amplitude (mV) & $8.7 \pm 6.1$ & $11.9 \pm 5.7$ & $\mathrm{p}=0.035$ \\
Impedance (ohms) & $615.3 \pm 209.8$ & $715.0 \pm 154.2$ & $\mathrm{p}=0.012$ \\
First follow up & & & \\
Days from implant & $43.4 \pm 38.3$ & $44 \pm 30.2$ & $\mathrm{p}=0.944$ \\
Threshold Amplitude (V @ 0.5 ms) & $1.4 \pm 1.046$ & $0.7 \pm 0.3$ & $\mathrm{p}<0.001$ \\
R-Wave Amplitude (mV) & $8.6 \pm 6.4$ & $16.0 \pm 4.9$ & $\mathrm{p}<0.001$ \\
Impedance (Ohms) & $454.0 \pm 118.4$ & $540.3 \pm 59.6$ & $\mathrm{p}<0.001$ \\
Last Follow-Up & & & \\
Days from implant & $398.8 \pm 259.3$ & $228 \pm 124.3$ & $\mathrm{p}<0.001$ \\
Paced QRS duration & $123.0 \pm 20.5$ & $116 \pm 16.1$ & $\mathrm{p}=0.186$ \\
Left Ventricular Ejection Fraction & $50.6 \pm 13.9$ & $55.5 \pm 13.1$ & $\mathrm{p}=0.207$ \\
Bipolar Pacing at Most Recent Follow-Up & $38 / 41(92.7 \%)$ & $43 / 43(100 \%)$ & $\mathrm{p}=0.071$ \\
Unipolar Pacing at Most Recent Follow-Up & $3 / 41(7.3 \%)$ & $0 / 43(0 \%)$ & $\mathrm{p}=0.071$ \\
Threshold Amplitude (V @ 0.5 ms) & $2.1 \pm 2.2$ & $0.8 \pm 0.3$ & $\mathrm{p}<0.001$ \\
R-Wave Amplitude (mV) & $8.90 \pm 6.6$ & $16.1 \pm 4.5$ & $\mathrm{p}<0.001$ \\
Impedance (Ohms) & $434.2 \pm 108.1$ & $535.5 \pm 56.8$ & $\mathrm{p}<0.001$ \\
Lead revision (\%) & $6 / 45(13.3 \%)$ & $0 / 42(0.0 \%)$ & $\mathrm{p}=0.014$ \\
\hline
\end{tabular}

Table 3: Acceptable Pacing Parameters of Patients Undergoing Physiologic Pacing

\begin{tabular}{llll}
\hline & His Pacing & LBBA Pacing & P-value \\
\hline Post-Implant & $22 / 32(68.8 \%)$ & $26 / 30(86.7 \%)$ & $\mathrm{p}=0.092$ \\
Post-Procedure Follow-Up & $14 / 25(56.0 \%)$ & $27 / 28(96.4 \%)$ & $\mathrm{p}=0.001$ \\
Most Recent Follow-Up & $17 / 28(60.7 \%)$ & $37 / 39(94.9 \%)$ & $\mathrm{p}=<0.001$ \\
\hline
\end{tabular}


Acceptable parameters are defined as sensing $>5 \mathrm{mV}$, threshold $<2.5 \mathrm{~V} @ 0.5 \mathrm{~ms}$ and impedance $<1200$ Ohms.

Proportion with Acceptable Pacing Endpoint

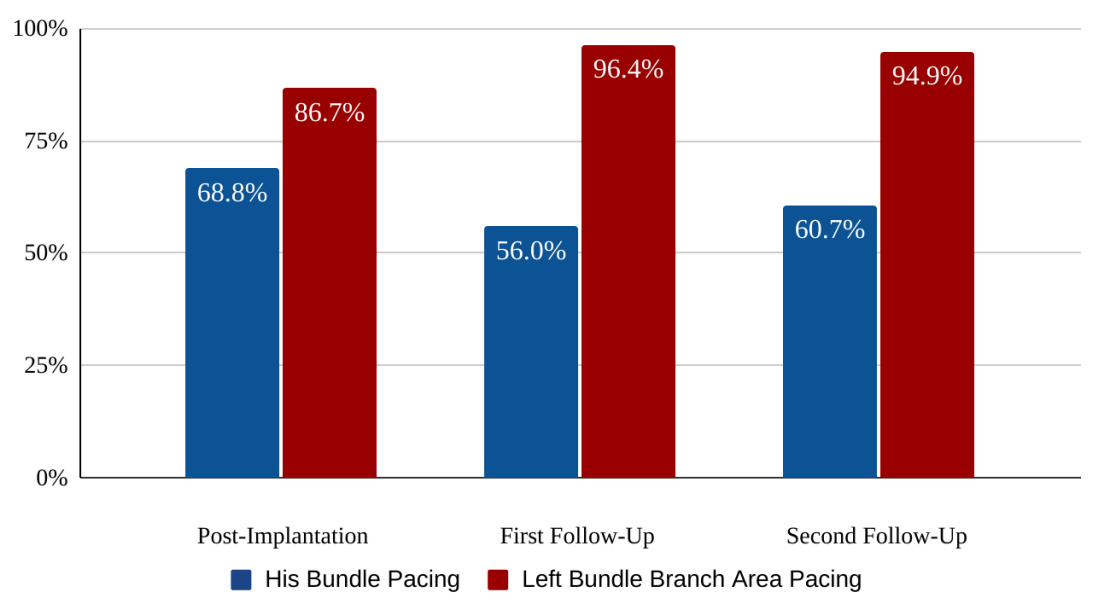

Figure 1. Percentage of patients receiving HBP and LBBAP who met the combined acceptable pacing endpoint (sensing $>5 \mathrm{mV}$, threshold $<2.5 \mathrm{~V} @ 0.5 \mathrm{~ms}$ and impedance $<1200 \mathrm{Ohms}$ ) at each interval followup.

Impedance Over Follow-Up

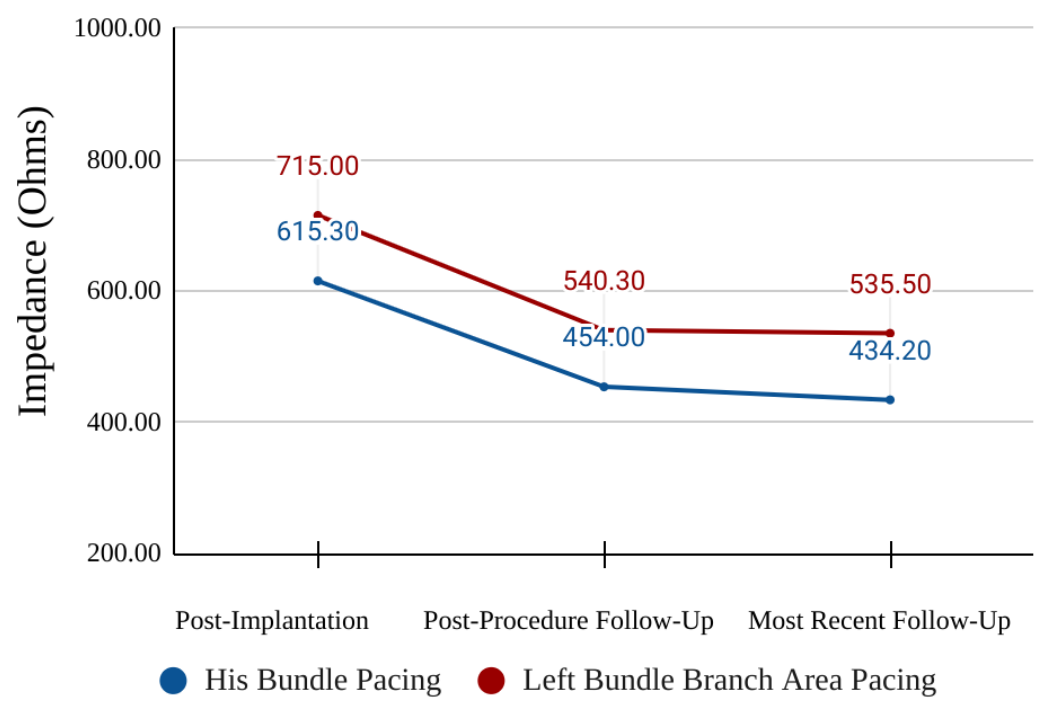




\section{R-Wave Amplitude Over Follow-Up}

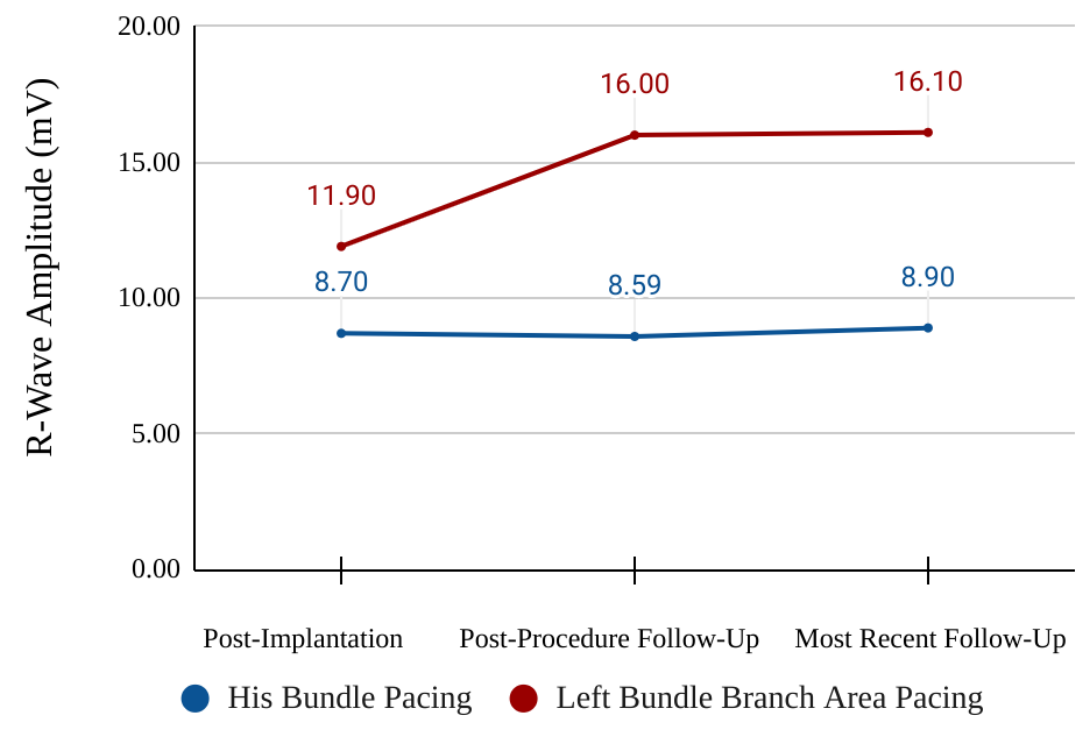

Pacing Thresholds Over Follow-Up

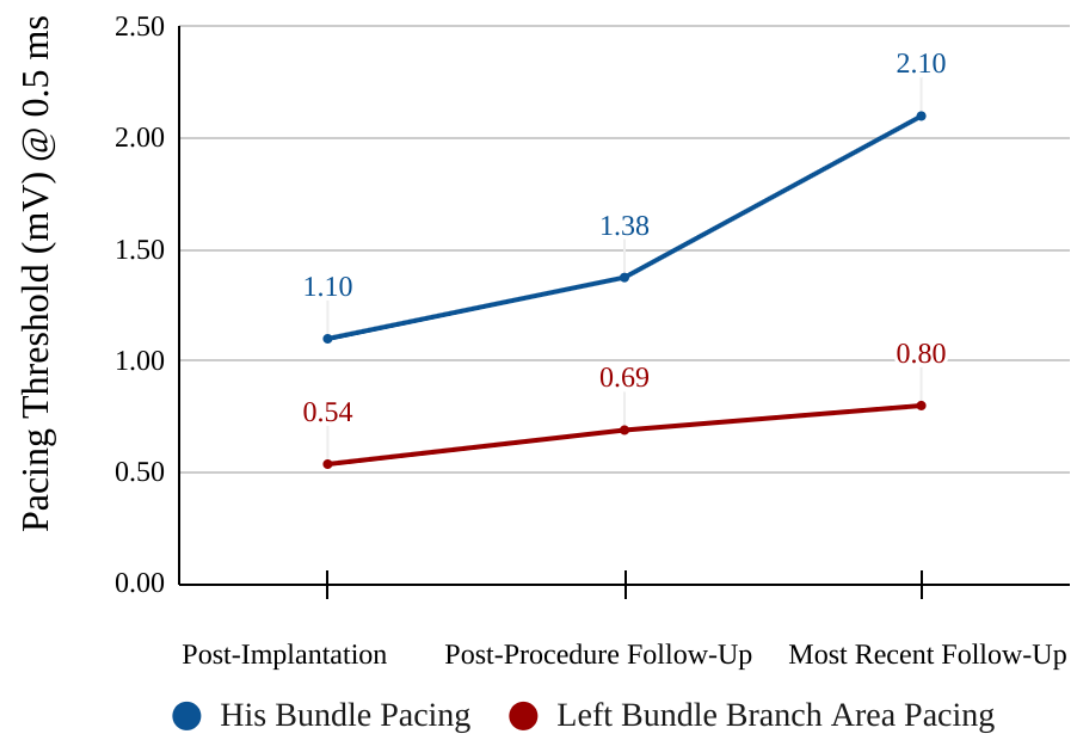

Figure 2. Pacing threshold, R-wave amplitude, impedance values of the HBP and LBBAP at three follow-up points. 


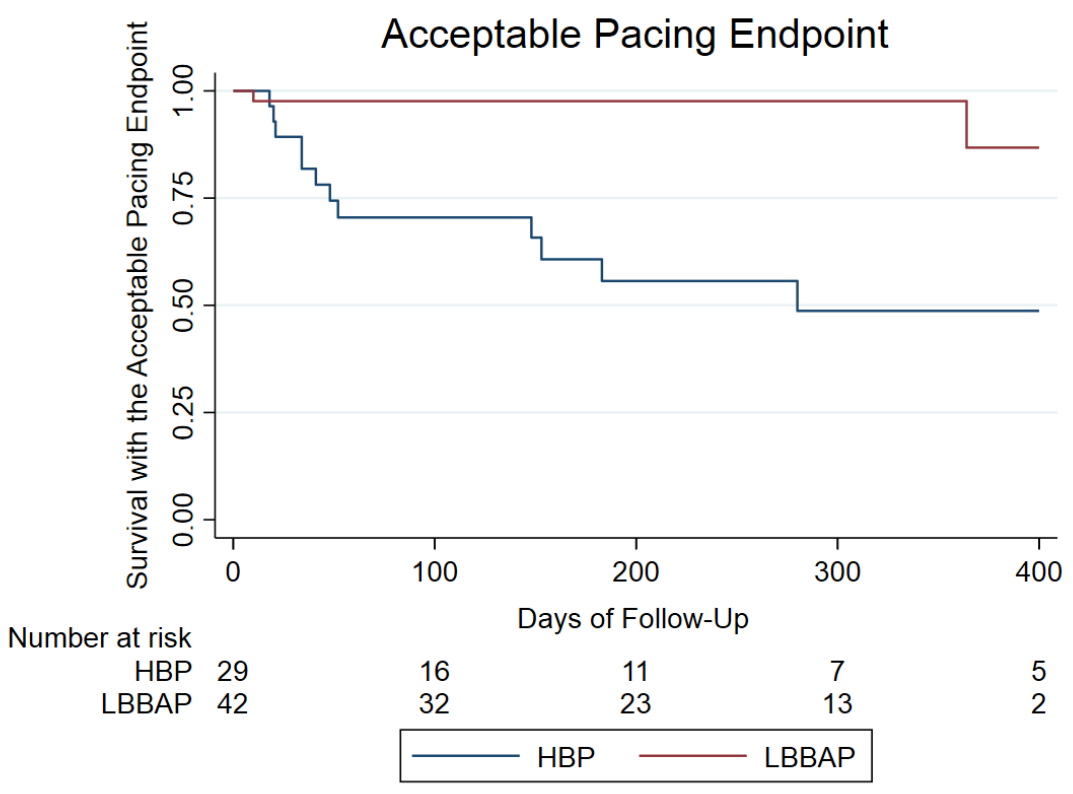

Figure 3. Kaplan-Meier survival analysis showing the incidence of the combined acceptable pacing endpoint (sensing $>5 \mathrm{mV}$, threshold $<2.5 \mathrm{~V} @ 0.5 \mathrm{~ms}$ and impedance $<1200 \mathrm{Ohms}$ ) in patients with HBP and LBBAP during follow-up.

\section{References}

1. Shukla HH, Hellkamp AS, James EA, et al. Heart failure hospitalization is more common in pacemaker patients with sinus node dysfunction and a prolonged paced QRS duration. Heart Rhythm. 2005; 2: $245-51$.

2. Sharma AD, Rizo-Patron C, Hallstrom AP et al. Percent right ventricular pacing predicts outcomes in the DAVID trial. Heart Rhythm. 2005; 2: 830-4.

3. Nielson JC, Kristensen L, Anderson HR et al. A randomized comparison of atrial and dual chamber pacing in 177 consecutive patients with sick sinus syndrome: echocardiographic and clinical outcome. J Am Coll Cardiol. 2003; 42: 614-623.

4. Sweeney MO, Hellkamp AS, Ellenberger KA, et al. Adverse effect of ventricular pacing on heart failure and atrial fibrillation among patients with normal baseline QRS duration in a clinical trial of pacemaker therapy for sinus node dysfunction. Circulation. 2003; 107 (23): 2932-2937.

5. Steinberg JS, Fischer A, Wang P, et al. The clinical implications of cumulative right ventricular pacing in the Multicenter Automatic Defibrillator Trial II. J Cardiovasc Electrophysiol. 2005; 16: 359-65

6. Deshmukh P, Casavant DA, Romanyshyn M and Anderson K. Permanent, direct Hi-bundle pacing: a novel approach to cardiac pacing in patients with normal His-Purkinje activation. Circulation. 2000; 101: 869-877.

7. Barba-Pichardo R, Morina-Vazquez P, Venegas-Gamero J, Maroto-Monserrat F et al. Permanent Hisbundle pacing in patients with infra-hisian atrioventricular block. Rev Esp Cardiol. 2006; 59: 553-558.

8. Upadhyay GA, Vijayaraman P, Nayak HM, Verma N et al. On-treatment comparison between corrective His bundle pacing and biventricular pacing for cardiac resynchronization: A secondary analysis of the His-SYNC pilot trial. Heart Rhythm. 2019; 16: 1797-1807.

9. Lustgarten DL, Crespo EM, Arkhipova-Jenkins I et al. His-bundle pacing versus biventricular pacing in cardiac resynchronization therapy patients: A crossover design comparison. Heart Rhythm. 2015; 12: $1548-1557$.

10. Sharma PS, Dandamudi G, Herweg B et al. Permanent His-bundle pacing as an alternative to biven- 
tricular pacing for cardiac resynchronization therapy: a multicenter experience. Heart Rhythm. 2018; 15: $413-420$.

11. Bhatt GA, Musat DL, Milstein N, Pimienta J et al. The efficacy of his bundle pacing: lessons learned from implementation for the first time at an experienced electrophysiology center. J Am Colle Cardiol EP. 2018; 4: 1397-1406.

12. Abdelrahman M, Subzposh FA, Beer D, Durr B et al. Clinical outcomes of his bundle pacing compared to right ventricular pacing. J Am Coll Cardiol. 2018; 71: 2319-2330.

13. Vijayaraman P, Naperkowski A, Subzposh FA, Abdelrahman M et al. Permanent his-bundle pacing: Long-term lead performance and clinical outcomes. Heart Rhythm. 2018; 15: 696-702.

14. Keene D, Arnold AD, Jastrzebski M, Burri H et al. His bundle pacing, learning curve, procedure characteristics, safety, and feasibility: Insight from a large international observational study. J Cardiovasc Electrophysiol. 2019; 30: 1984-1993.

15. Huang W, Chen X, Su L, Wu S, Xia X, Vijayaraman P. A beginner's guide to permanent left bundle branch pacing. Heart Rhythm. 2019; $16:$ 1791-1796.

16. Vijayaraman P, Subzposh FA, Naperkowski A, Panikkath R et al. Prospective evaluation of feasibility and electrophysiologic and echocardiographic characteristics of left bundle branch area pacing. Heart Rhythm. 2019; 16: 1774-1782.

17. Guo J, Li L, Meng F, Su M, Huang X, Chen S, Li Q, Chang D, Cai B. Short-term and intermediateterm performance and safety of left bundle branch pacing. J Cardiovasc Electrophysiol. 2020; 31 (6): $1472-1481$

18. Ponnusamy SS, Muthu G, Kumar M, Bopanna D, Anand V, Kumar S. Mid-term feasibility, safety and outcomes of left bundle branch pacing-single center experience. J Interv Card Electrophysiol. 2021; 60: 337-346.

19. Chen K, Li Y, Dai Y, et al. Comparison of electrocardiogram characteristics and pacing parameters between left bundle branch pacing and right ventricular pacing in patients receiving pacemaker therapy. Europace. 2019; $21: 673-680$.

20. Padala SK and Ellenbogen KA. Left bundle branch pacing is the best approach to physiological pacing. Heart Rhythm O2. 2020; $1:$ 59-67.

21. Peng H, Sun Z, Zhang H, Ma W. Long-term performance of right ventricular pacing leads: risk factors associated with permanent right ventricular pacing threshold increase. J Interv Card Electrophysiol. 2019; $55: 349-357$

22. Liu X, Gu M, Hua W, Hu Y, Niu HX, Cai M, Zhang N, Zhang S. Comparison of electrical characteristics and pacing parameters of pacing different parts of the His-Purkinje system in bradycardia patients. J Interv Card Electrophysiol. doi: 10.1007/s10840-021-00962-8.

23. Molina-Lerma M, Macías-Ruiz R, Sánchez-Millán P, Jiménez-Jáimez J, Tercedor-Sánchez L, Álvarez M. Comparative analysis of His-bundle pacing and left bundle branch area pacing: acute and short-term results. Rev Esp Cardiol (Engl Ed). 2021; 74: 628-630.

24. Teigeler T, Kolominsky J, Vo C, Shepard RK, Kalahasty G, Kron J, Huizar JF, Kaszala K, Tan AY, Koneru JN, Ellenbogen KA, Padala SK. Intermediate-term performance and safety of His-bundle pacing leads: A single-center experience. Heart Rhythm. 2021; $18: 743-749$.

25. Chaumont C, Auquier N, Milhem A, Mirolo A, Al Arnaout A, Popescu E, Viart G, Godin B, Gillibert A, Savouré A, Eltchaninoff H, Anselme F. Can permanent His bundle pacing be safely started by operators new to this technique? Data from a multicenter registry. J Cardiovasc Electrophysiol. 2021; $32: 417-427$.

26. Qian Z, Qiu Y, Wang Y, Jiang Z, Wu H, Hou X, Zou J. Lead performance and clinical outcomes of patients with permanent His-Purkinje system pacing: a single-centre experience. Europace. doi: 10.1093/europace/euaa295.

27. Mittal S, Bhatt AG. Left Bundle Branch Pacing: A Contender Emerges to Challenge the 25-Year Run of Cardiac Resynchronization Therapy. JACC Clin Electrophysiol. 2020; 6: 859-862.

28. Su L, Wang S, Wu S, Xu L, Huang Z, Chen X, Zheng R, Jiang L, Ellenbogen KA, Whinnett ZI, Huang W. Long-Term Safety and Feasibility of Left Bundle Branch Pacing in a Large Single-Center Study. 
Circ Arrhythm Electrophysiol. 2021; 14 : e009261.

29. Chen X, Jin Q, Bai J, Wang W, Qin S, Wang J, Liang Y, Su Y, Ge J. The feasibility and safety of left bundle branch pacing vs. right ventricular pacing after mid-long-term follow-up: a single-center experience. Europace. doi: 10.1093/europace/euaa294.

30. Salden FCWM, Luermans JGLM, Westra SW, Weijs B, Engels EB, Heckman LIB, Lamerichs LJM, Janssen MHG, Clerx KJH, Cornelussen R, Ghosh S, Prinzen FW, Vernooy K. Short-Term Hemodynamic and Electrophysiological Effects of Cardiac Resynchronization by Left Ventricular Septal Pacing. J Am Coll Cardiol. 2020;75 (4): 347-359. 